\title{
ADAPTABILIDADE DE CARREIRA: PRODUÇÃO CIENTÍFICA DOS ÚLTIMOS 10 ANOS
}

\section{CAREER ADAPTABILITY: SCIENTIFIC PRODUCTION OF THE LAST 10 YEARS}

\author{
JUCIÉLE FÁTIMA CORADINI
}

Universidade Federal de Santa Maria (UFSM)

Mestra em Administração - PPGA/UFSM

Orcid: http://orcid.org/0000-0003-3607-3052 / E-mail: jucielecoradini@gmail.com

\section{LUIS FELIPE DIAS LOPES}

Professor Titular na Universidade Federal de Santa Maria (UFSM)

Doutor em Engenharia de Produção (PPGEP/UFSC)

Orcid: http://orcid.org/0000-0001-6756-9179 / E-mail: Iflopes67@yahoo.com.br

Rua Dona Luiza, 216 (casa), Santa Maria, RS

\section{ADRIANE FABRICIO}

Universidade Federal de Santa Maria (UFSM)

Doutora em Administração (PPGA/UFSM)

Orcid: http://orcid.org/0000-0002-3508-2769 / E-mail: adrianefabricio@yahoo.com.br

\section{MAUREN PIMENTEL LIMA}

Universidade Federal de Santa Maria (UFSM)

Mestra em Administração - PPGA/UFSM

Orcid: http://orcid.org/0000-0002-0215-1190 / E-mail: maurenlima@hotmail.com

\section{CRISTIANE KRÜGER}

Universidade Federal de Santa Maria (UFSM)

Doutora e Mestra em Administração - PPGA/UFSM

Orcid: http://orcid.org/0000-0003-2271-6432 / E-mail: cristiane.kruger@ufsm.br

Submissão: 24/06/2020. Revisão: 20/12/2021. Aceite: 17/01/2022. Publicação: 01/02/2022 .

DOI: http://dx.doi.org/10.22277/rgo.v15i1.5606

\section{RESUMO}

Objetivo: Avaliar a produção científica sobre adaptabilidade de carreira dos últimos 10 anos, no período de 2010-2019.

Método / Abordagem: Realizou-se uma revisão bibliométrica da literatura nos bancos de dados Web of Science e Scopus, entre os meses de janeiro a fevereiro de 2020, resultando, respectivamente, na seleção de 390 e 417 publicações. As publicações foram analisadas em função do ano, área, autor, fonte, país, tema e hot topics.

Principais resultados: Verificou-se que em ambas as bases pesquisadas há concentração de publicações quanto ao tema no ano de 2019, nos Estados Unidos da América, sendo os hot topics Desenvolvimento da Carreira e Empregabilidade, principalmente na área de Psicologia.

Este é um artigo publicado em acesso aberto (Open Access) sob a licença Creative Commons Attribution, que permite uso, distribuição e reprodução em qualquer meio, sem restrições desde que o trabalho original seja corretamente citado. 
Originalidade / Relevância: O uso da bibliometria vem a ser uma ferramenta de busca e disseminação do conhecimento científico, possibilitando aos pesquisadores uma análise quantitativa e prognóstica das publicações realizadas em determinadas áreas científicas.

Palavras-chave: Carreira. Adaptabilidade. Bibliometria.

\section{ABSTRACT}

Purpose: Evaluate the scientific production on Career Adaptability over the ten-year period of 2010 to 2019

Method / Approach: A bibliometric review of the literature was conducted in the Web of Science and Scopus databases between January and February 2020, resulting respectively in the selection of 390 and 417 publications. These publications were analyzed according to the year, area, author, source, country, theme and hot topics.

Main Findings: it was observed that in both bases were concentrations of publications on the subject in 2019 in the United States of America, and the hot topics were career development and employability, mainly in the field of psychology.

Originality / Relevance: The use of bibliometrics is a tool for the search and dissemination of scientific knowledge, enabling researchers to perform a quantitative and prognostic analysis of publications in certain scientific areas.

Keywords: Career. Adaptability. Bibliometrics.

\section{INTRODUÇÃO}

O conceito de adaptabilidade de carreira se refere à capacidade dos indivíduos em utilizar suas habilidades para lidar com tarefas, transições ou traumas oriundos de seus papéis sociais, o que pode ocorrer de maneira antecipatória ou situacional (Savickas \& Porfeli, 2012). Já a ocupação laboral, no entendimento de Souza e Correa (2016), deve ser entendida não só como uma realização mecânica, mas como a representação do status no meio em que o sujeito está inserido. Nessa perspectiva, para os mesmos autores, o trabalho assume diferentes conotações, sendo marcado por uma característica paradoxal, onde pode, simultaneamente, assumir uma perspectiva otimista e benéfica, como também ser algo prejudicial representando, uma forma de controle social que pode promover o sofrimento humano.

A evolução da carreira é um processo que está ligado à trajetória de vida individual, a qual inclui seu percurso profissional. Trata-se de um processo construído de forma dinâmica, incluindo elementos que estão associados à subjetividade, inter-relacionados aos eventos individuais e da construção histórica institucional (Zanelli, Silva \& Tordera, 2013). Nesse sentido, o sujeito torna-se o protagonista de mudanças, tanto para ele quanto para a comunidade. A descentralização contribui para a valorização das características, tais como: flexibilidade, autonomia, criatividade e iniciativa no planejamento de carreira (Magalhães, \& Berdassolli, 2013). Assim, é possível afirmar que a construção da carreira leva em conta, além dos objetivos e eventos individuais, a estrutura organizacional.

No que se refere às organizações, a descoberta mais relevante ao longo dos anos, nos estudos de Dutra (2017), foi a de que as trajetórias de carreira não possuem alterações ao longo do tempo, ou seja, se mantém estáveis, servindo como referência norteadora do desenvolvimento profissional dos indivíduos e importante ferramenta de gestão das organizações. Com base em avaliações, os esforços dos estudos sobre carreira têm buscado 
a construção de modelos teóricos mais gerais, voltados ao maior entendimento e ampliação do processo que envolve o indivíduo com a sua escolha e comprometimento de carreira (Carvalho, 2007).

Tendo em vista o exposto, o planejamento de carreira no Brasil vem ganhando importância e a sua prática tem sido iniciada por meio da avaliação pessoal, com o intuito de descobrir as habilidades e competências que o indivíduo possui, quais podem ser desenvolvidas ou as crenças limitantes que podem ser afastadas, de modo a realizar melhores escolhas na carreira, mesmo frente às incertezas do mercado de trabalho (Dias \& Soares, 2012). Outra observação destes autores refere-se à transição entre a conclusão da graduação e entrada dos indivíduos no mercado de trabalho, onde seu percurso pode apresentar possibilidades ou riscos, levando os formandos, de um modo geral, a fazerem suas escolhas permeadas pela angústia quanto ao futuro incerto de suas decisões.

Outra questão importante a ser destacada é a pandemia instalada pelo novo Coronavírus (COVID-19) e a crise econômica e social advinda desta, que tem impactado fortemente as mais diversas esferas de vida das populações (FVG, 2020). Por outro lado, os mesmos especialistas defendem que o atual momento de crise tornou ainda mais evidentes e necessárias algumas habilidades, como a resiliência e a capacidade de adaptação dos profissionais, acarretando mudanças irreversíveis quanto às formas de atuação (FGV, 2020).

Tendo em vista que os profissionais que estão buscando uma oportunidade de emprego necessitam de adaptação na carreira, e os que estão em atividade podem se apresentar satisfeitos ou não com o seu trabalho, devido à combinação dos recursos e do envolvimento nas atividades que desempenham, bem como na realização profissional, insere-se um contexto de reflexão sobre a problemática ser estudada. Em face dessas considerações, este estudo será guiado pelo seguinte problema de pesquisa: o que existe de novo e conceitual sobre pesquisas envolvendo adaptabilidade de carreira?

O presente estudo tem como objetivo avaliar a produção científica sobre a adaptabilidade de carreira nos últimos 10 anos (2010-2019), que vem a ser a capacidade das pessoas em se adaptarem e lidarem com as possíveis alterações na trajetória de carreira, nas principais bases de artigos científicos, Web of Science e Scopus.

Sabe-se que a temática adaptabilidade de carreira engloba habilidades que são vitais para o sucesso dos futuros profissionais e que, no momento, estes convivem com incertezas que desencadeiam medo ou até mesmo resistência para aceitar as mudanças. Por isso, flexibilização e adaptação são competências cada vez mais valorizadas no mercado de trabalho, que está em constante transformação, considerando atualmente a digital e, mais especificamente, a era home office (Helal, 2005; Alves \& Miranda, 2019).

Para isso, é necessário quebrar paradigmas, desafiar os limites e mudar a forma de agir, encontrando maneiras mais eficientes, seguras e rápidas para adaptar-se ao mundo em transformação. Também, desenvolver a adaptabilidade, que vem a ser a capacidade de mudar e tornar-se flexível. Pessoas naturalmente curiosas e corajosas se adaptam facilmente à flexibilização, para tanto, saem na frente em busca da adaptabilidade, mas isso não quer dizer que os indivíduos não conseguem aprimorar essa habilidade, pois todo o indivíduo que quer enfrentar o mercado de trabalho deve estar disposto a superar suas limitações e vivenciar o novo.

É preciso assumir o risco de mudar, se não decidirmos mudar, alguém estará decidindo em nosso lugar. Portanto, não devemos ter medo de assumir as rédeas do destino, para isso, nunca devemos parar de aprender, crescer, adquirir novos conhecimentos e ser resiliente, isso é muito importante para a formação de uma carreira vencedora (Moura 
\& Tomei, 2021). Assim, na atual situação que vivemos, a adaptabilidade na carreira é fundamental, pois é a partir dela que nos tornamos mais competitivos para o mercado de trabalho (Oliinyk et al., 2020).

\section{CARREIRA E ADAPTABILIDADE}

O conjunto de influências recebidas pelo indivíduo no meio em que está inserido tem sido amplamente estudado, com base em sua socialização, momento atual e profissional, gestão da própria carreira, livre arbítrio nas organizações e no mercado de trabalho (Dutra, 2018). O mesmo autor ressalta que, as pessoas em geral, percebem as oportunidades de trabalho e de carreira do meio externo para o interno, ou seja, consideram primeiramente o mercado e a empresa, buscando oportunidades profissionais, sem levar em conta as suas características individuais, desejos e sonhos, ocasionando a possibilidade de alguns equívocos, como armadilhas profissionais, desconforto profissional, e visão restrita de oportunidades.

Dessa forma, o planejamento de carreira tem se tornado essencial para tomada de decisões e para o desempenho profissional, o qual é composto por um conjunto de ações estruturadas que visam resultados melhores do indivíduo com a empresa e a comunidade. Tal planejamento permite que os indivíduos obtenham decisões acertadas no presente, com boas implicações futuras (Petska, 2015).

A imprevisibilidade da carreira existente passou a ser reforçada com o avanço tecnológico e o advento da globalização nas sociedades, o que passou a exigir o desenvolvimento de novas habilidades dos profissionais, comparadas às exigidas no século passado (Fiorini, Bardagi \& Silva, 2016). Como consequência, um novo posicionamento passou a ser exigido pelos profissionais contemporâneos no mundo do trabalho. As decisões profissionais não ficam mais restritas somente ao momento da escolha profissional, compreendem, também, desde o momento de inserção do profissional no mercado de trabalho, sua permanência, seu desenvolvimento, transição de carreira, desligamento, até sua aposentadoria, exigindo do mesmo reflexões contínuas e reposicionamento de carreira durante toda sua vida profissional (Brasil et al., 2012).

Savickas e Porfeli (2012) abordam o conceito de adaptabilidade de carreira como a capacidade dos indivíduos em utilizar suas habilidades para lidar com tarefas, transições e/ou traumas em seus papéis sociais, de forma antecipatória ou situacional. Além disso, os autores evidenciam que o assunto vem ganhando força. Dentre as habilidades adquiridas, são destacadas como principais: preocupação, controle, curiosidade e confiança (Savickas \& Porfeli, 2012).

A seguir, apresenta-se o método utilizado para a realização da pesquisa bibliométrica nas bases de dados Web of Science (WoS) e Scopus, no período de 2010 a 2019.

\section{ASPECTOS METODOLÓGICOS}

Trata-se de uma pesquisa bibliométrica, de caráter descritivo e abordagem quantitativa. Para Wingerter et al. (2018) a pesquisa bibliométrica é composta por leis e princípios empíricos, cujo objetivo é explorar os aspectos quantitativos encontrados. Lopes et al. (2012) complementam que a bibliometria e seus indicadores são utilizados para avaliar a qualidade dos periódicos científicos, avaliar a produtividade dos autores e realizar estudos de citações.

Para Ferenhof e Fernandes (2015) algumas das etapas básicas para a realização da pesquisa bibliométrica são: definição da base da pesquisa, que determinará os indicadores; a 
base de dados a ser utilizada e a forma como os documentos serão selecionados e padronizados; a consolidação e a análise das informações obtidas; e, a síntese e a elaboração de relatórios. Corroborando com estes autores, Pimenta, Portella, Oliveira e Ribeiro (2017) descrevem que a bibliometria seria uma permuta ou troca de informações, apresentando algumas de suas características: indicar os estudos em crescimento, constatar a diminuição de determinados temas, medir o impacto das revistas científicas, mensurar as instituições de ensino e autores que possuem maior produção acadêmica.

Tendo em vista tais informações, realizou-se uma pesquisa bibliométrica objetivando ampliar o conhecimento referente às publicações sobre o tema "Career Adaptability", nas bases Web of Science (WoS) e Scopus, por serem as bases mais consultadas no contexto acadêmico, no período de 2010 a 2019.

A primeira etapa constituiu-se da busca pelas expressões, de forma isolada com as palavras chaves "Career Adaptability", com o objetivo de obter as características gerais da produção científica, como áreas temáticas, publicações por ano, títulos de fontes, autores, países, idiomas e temas mais relevantes, os hot topics. Na segunda etapa foram identificados os principais tópicos escolhidos e relacionados à temática, sendo elencados os temas.

$\mathrm{Na}$ terceira etapa foram apresentados os hot topics, que são os tópicos mais relevantes de cada tema, por meio da identificação dos índices $\mathrm{h}$ e $\mathrm{h}$-b. Conforme Banks (2006), criador do índice h-b, seria uma extensão do h-index, desenvolvido por Jorge Hirsch para quantificar as publicações de um autor, já que o índice h-b é obtido por meio do número de citações de um tópico ou da combinação em determinado período, listados em ordem decrescente das citações.

Em relação ao índice " $m$ ", os valores entre $0<m \leq 0,5$ podem ser classificados como de interesse para pesquisadores em um campo específico de pesquisa, o qual engloba uma comunidade pequena; o índice $0,5<m \leq 2$ provavelmente pode se tornar um hot topic, como na área de pesquisa, na qual a comunidade é maior ou o tópico apresenta características muito interessantes; por fim, o índice $m>2$ é considerado um hot topic, tópico exclusivo, com alcance não apenas na sua própria área de pesquisa, e é provável que tenha efeitos de aplicação ou característica únicas (Banks, 2006).

$O$ autor explica que para encontrá-lo divide-se o índice $h-b$ pelo período da pesquisa estipulado na base de dados. Objetivando analisar esses índices, utilizou-se as seguintes definições de Banks (2006):

Índice $\mathrm{h}$ - $\mathrm{b}$ = número de citações de um autor em um determinado período.

$$
m=\frac{\text { índice } h-b}{10}
$$

Nesta pesquisa foram selecionados 12 tópicos relacionados à temática, de acordo com o número de publicações, sendo tópicos quentes somente os que possuírem o índice $\mathrm{m}$ $>2$.

\section{ANÁLISE DAS BUSCAS NAS BASES WOS E SCOPUS}

Nesta seção serão apresentados os principais resultados relativos ao estudo bibliométrico, realizado sobre o tema Adaptabilidade de carreira na última década. Para tanto, foram analisadas as 12 primeiras posições deste tema e classificadas por maior 
número de registros (área temática, publicações por ano e fonte) e maior número de citações do artigo (autores) sobre a temática.

A partir da inserção do descritor "Career adaptability", foram encontradas na base WoS 390 publicações e na Scopus 417 publicações, no período de 2010 a 2019 (Figura 1), para abrangência do assunto pesquisado e para fins de evolução entre as duas bases de pesquisa. Dessas, foram selecionadas as dez mais relevantes.

Figura 1

Evolução das publicações por ano do tema Adaptabilidade de carreira

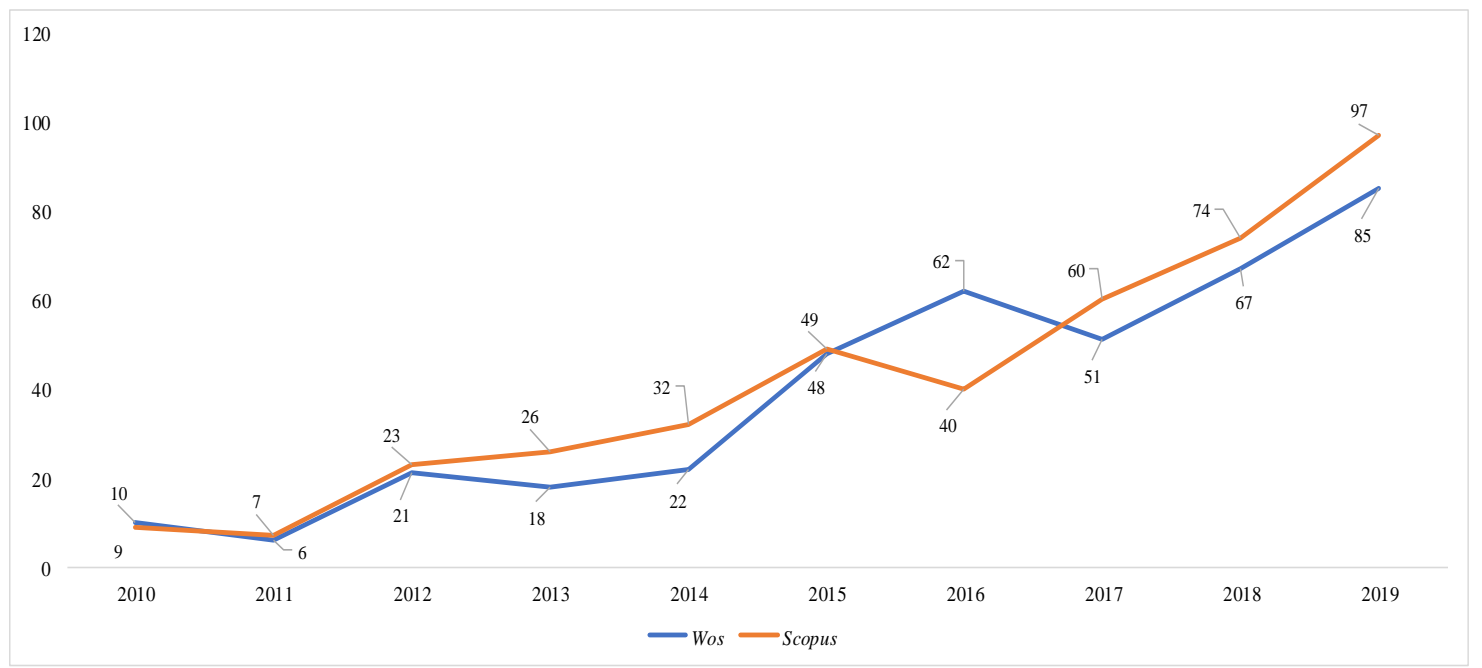

Fonte: elaborado com base em Web of Science e Scopus (2020).

Conforme a Figura 1, pode-se observar um aumento linear em ambas as bases, havendo destaque para o ano de 2019, o qual teve o maior número de publicações sobre o tema Adaptabilidade de carreira. Na Tabela 1 são apresentadas as dez principais áreas temáticas relacionadas à Adaptabilidade de carreira e o seu respectivo número de publicações.

Tabela 1

Número de publicações do tema Adaptabilidade de carreira por área nas bases pesquisadas

\begin{tabular}{c|c|c|c}
\hline \multicolumn{2}{c|}{ WoS } & \multicolumn{2}{c}{ Scopus } \\
\hline Áreas de Pesquisa & Registro (\%) & Áreas de Pesquisa & Registro (\%) \\
\hline PSYCHOLOGY & $304(77,95)$ & PSYCHOLOGY & $312(41,4)$ \\
BUSINESS ECONOMICS & $45(11,54)$ & $\begin{array}{c}\text { BUSINESS, MANAGEMENT } \\
\text { AND ACCOUNTING }\end{array}$ & $218(28,9)$ \\
EDUCATION EDUCATIONAL & $45(11,54)$ & SOCIAL SCIENCES & $178(23,6)$ \\
RESEARCH & & ECONOMICS, & \\
SOCIAL SCIENCES OTHER & $13(3,33)$ & ECONOMETRICS AND & $24(3,2)$ \\
TOPICS & $3(0,76)$ & FINANCES & $15(2,0)$ \\
ENGINEERING & $3(0,76)$ & MRTSICINE AND HUMANITIES & $3(0,4)$ \\
ENVIRONMENTAL SCIENCES & $3(0,76)$ & NURSING & $3(0,4)$ \\
ECOLOGY & $3(0,76)$ & NEUROSCIENCE & $1(0,1)$ \\
MATHEMATICS & $3(0,76)$ & & \\
NURSING & $3(0,76)$ & & \\
REHABILITATION & & \\
SCIENCE TECHNOLOGY & & \\
OTHER TOPICS & & & \\
\hline
\end{tabular}

Fonte: Web of Science e Scopus (2020). 
$\mathrm{Na}$ Tabela 1, verifica-se que a área com maior destaque na WoS é a Psicologia (77,95\%), seguida de Educação de Negócios Econômicos e da Pesquisa Educacional, ambos com 11,54\%. Na Scopus, a área com maior percentual de publicações também é a Psicologia (41,4\%), seguida de Negócios Gestão e Contabilidade (28,9\%) e das Ciências sociais $(23,6 \%)$. Com base na pesquisa nas bases WoS e Scopus, percebe-se que a temática é bastante discutida na área da saúde.

Na Figura 2 são apresentadas as publicações por autor, sobre o tema Adaptabilidade de carreira, nas bases de dados WoS e Scopus.

Figura 2

Publicações por autor sobre o tema Adaptabilidade de carreira nas bases pesquisadas

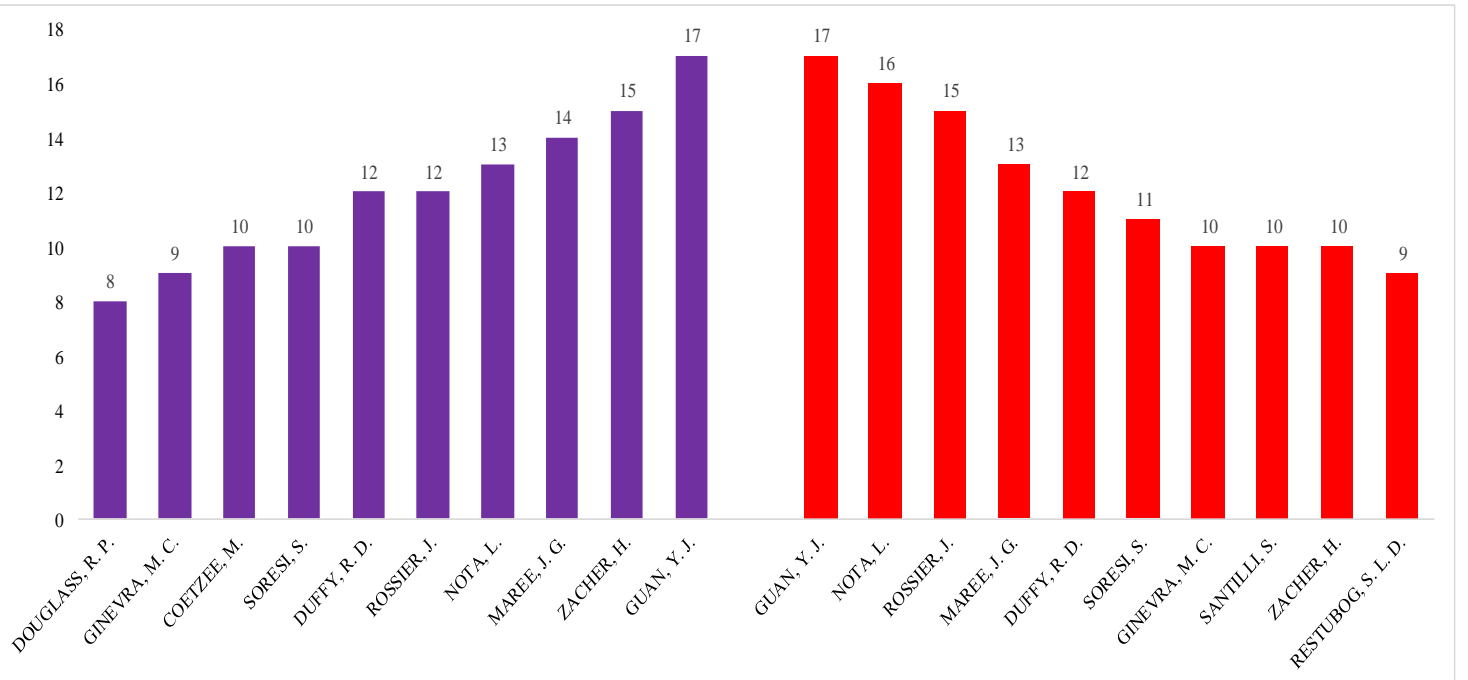

Fonte: Web of Science e Scopus (2020).

Por meio da Figura 2 observa-se que na WoS as maiores concentrações de publicações por autor, sobre Adaptabilidade de carreira, foram de Yanjun Guan (17) e Hannes Zacher (15), respectivamente. O autor Yanjun Guan é de nacionalidade chinesa, obteve seu PhD em Psicologia pela Universidade Chinesa de Hong Kong em 2009, e atualmente trabalha como professor de Administração em Durham University Business School, no Reino Unido. Seus interesses de pesquisa são sobre adaptabilidade na carreira, tomada de decisões na carreira, sucesso na carreira, ajuste pessoa-ambiente, interesses e valores vocacionais, bem como diferenças culturais no comportamento vocacional.

No que se tange à quantidade de publicações por autor na Scopus, destacam-se Yanjun Guan (17), o mesmo autor que lidera o ranking na WoS sobre o tema, seguido de Laura Nota (16). A autora Laura Nota é professora de Aconselhamento Psicológico para a Inclusão de Deficiências e Desconforto Social e de Construção de Carreira e Aconselhamento de Carreira no Departamento de Filosofia, Sociologia, Educação e Psicologia Aplicada. Também, é diretora do Curso de Mestrado, na Pós-Graduação em Inclusão e Inovação Social (2018 / 2019) da Universidade de Padova, na Itália.

A seguir, na Tabela 2, são apresentadas as principais fontes sobre o tema Adaptabilidade de carreira. 
Tabela 2

Principais fontes de Publicações do tema Adaptabilidade de carreira

\begin{tabular}{|c|c|c|c|}
\hline \multicolumn{2}{|l|}{ WoS } & \multicolumn{2}{|l|}{ Scopus } \\
\hline Título das fontes & Registro & Título das fontes & Registro \\
\hline JOURNAL OF AFFECTIVE & 438 & JOURNAL OF VOCATIONAL & 108 \\
\hline JOURNAL OF ANXIETY DISORDERS & 352 & JOURNAL OF CAREER ASSESSMENT & 29 \\
\hline DEPRESSION AND ANXIETY & 248 & $\begin{array}{l}\text { JOURNAL OF CAREER } \\
\text { DEVELOPMENT }\end{array}$ & 23 \\
\hline PSYCHIATRY RESEARCH & 226 & $\begin{array}{l}\text { CAREER DEVELOPMENT } \\
\text { QUARTERLY }\end{array}$ & 18 \\
\hline PLOS ONE & 204 & FRONTIERS IN PSYCHOLOGY & 11 \\
\hline $\begin{array}{l}\text { BEHAVIOUR RESEARCH AND } \\
\text { THERAPY }\end{array}$ & 191 & $\begin{array}{l}\text { JOURNAL OF PSYCHOLOGY IN } \\
\text { AFRICA } \\
\text { INTERNATIONAL JOURNAL FOR }\end{array}$ & 10 \\
\hline BIOLOGICAL PSYCHIATRY & 157 & $\begin{array}{l}\text { EDUCATIONAL AND VOCATIONAL } \\
\text { GUIDANCE }\end{array}$ & 8 \\
\hline PSYCHOLOGICAL MEDICINE & 154 & $\begin{array}{l}\text { REVISTA BRASILEIRA DE } \\
\text { ORIENTAÇÃO PROFISSIONAL }\end{array}$ & 7 \\
\hline BMC PSYCHIATRY & 151 & $\begin{array}{l}\text { CAREER DEVELOPMENT } \\
\text { INTERNATIONAL }\end{array}$ & 5 \\
\hline COGNITIVE THERAPY AND & 122 & & \\
\hline RESEARCH & $12 \angle$ & & \\
\hline
\end{tabular}

Fonte: Web of Science e Scopus (2020).

Visualiza-se, por meio da Tabela 2, que na base WoS o periódico com maior publicação é o Journal of Affective Disorders, com 438 publicações, seguido do Journal of Anxiety Disorders, com 352 publicações. Na base Scopus o periódico com maior número de publicações é o Journal of Vocational Behavior, representando em torno de 108 publicações, seguido do Journal of Career assessment, representando 29 publicações. Observa-se que ambos os jornais que ficaram no ranking da Scopus são os mesmos da WoS. O Journal of Vocational Behavior publica artigos empíricos e teóricos que contribuem para os campos de escolha de carreira, no desenvolvimento de carreira, aconselhamento e ajuste de trabalho ao longo da vida.

A seguir, apresentam-se os principais tipos de documentos sobre o tema Adaptabilidade de carreira (Tabela 3).

Tabela 3

Principais Tipos de documento sobre o tema Adaptabilidade de carreira

\begin{tabular}{c|c|c|c}
\hline \multicolumn{2}{c|}{ WoS } & \multicolumn{2}{c}{ Scopus } \\
\hline Tipos de Documentos & Registros & Tipos de Documentos & Registros \\
\hline ARTICLE & 353 & ARTICLE & 41 \\
PROCEEDINGS PAPER & 21 & BOOK CHAPTER & 6 \\
MEETING ABSTRACT & 12 & BOOK & 6 \\
EARLY ACCESS & 10 & CONFERENCE PAPER & 5 \\
REVIEW & 4 & REVIEW & 2 \\
EDITORIAL MATERIAL & 3 & EDITORIAL & 2 \\
BOOK CHAPTER & 1 & NOTE & 1 \\
& & CONFERENCE REVIEW & 10 \\
\hline
\end{tabular}

Fonte: Web of Science e Scopus (2020) 
Ao analisar a Tabela 3, percebe-se que tanto na WoS quanto na Scopus, o tipo de documento com mais registros sobre o tema em estudo são os artigos, totalizando 353 e 108 registros, respectivamente. Destaca-se que a maior parte desses estudos são trabalhos teóricos e empíricos resultantes de pesquisas que podem possuir alguma aplicação.

Quanto aos principais países com publicações sobre o tema Adaptabilidade de carreira, eles estão elencados por meio da Tabela 4 e Figura 3.

Tabela 4

Principais países com publicações sobre o tema Adaptabilidade de carreira

\begin{tabular}{c|c|c|c}
\hline País & WoS & País & Scopus \\
\hline ESTADOS UNIDOS & 95 & ESTADOS UNIDOS & 102 \\
CHINA & 72 & CHINA & 40 \\
AUSTRALIA & 48 & AUSTRÁLIA & 37 \\
AFRICA DO SUL & 33 & REINO UNIDO & 36 \\
INGLATERRA & 32 & ÁFRICA DO SUL & 28 \\
ALEMANHA & 23 & ITÁLIA & 26 \\
ITALIA & 23 & SUIÇA & 21 \\
PERU & 23 & ALEMANHA & 19 \\
PAISES BAIXOS & 21 & PERU & 18 \\
SUIÇA & 21 & PAÍSES BAIXOS & \\
\hline
\end{tabular}

Fonte: Web of Science e Scopus (2020).

Figura 3

Distribuição das publicações por países conforme as bases pesquisadas
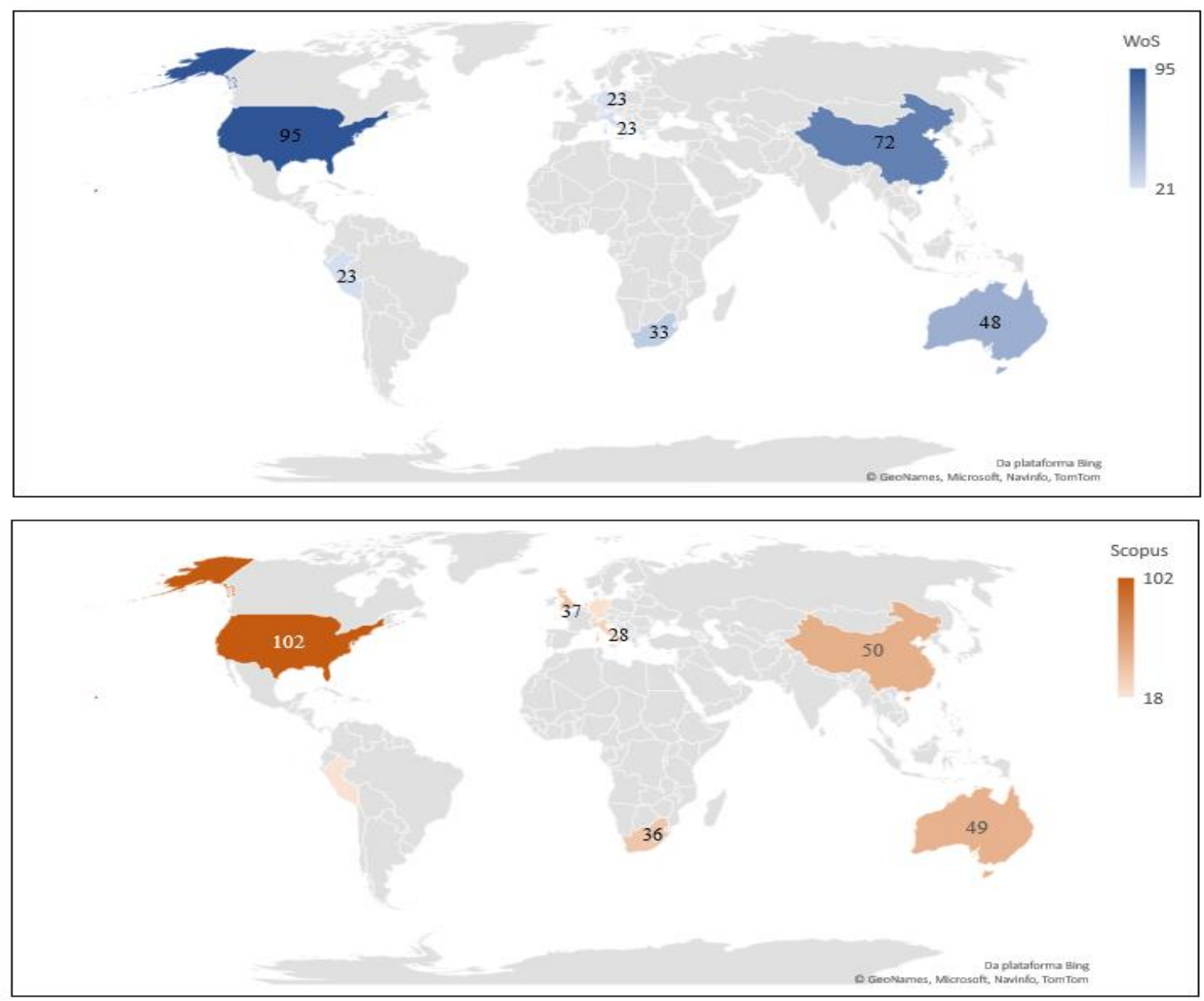

Fonte: Web of Science e Scopus (2020). 
Com base nos dados da Tabela 4 e da Figura 3, percebe-se que tanto na base WoS como na Scopus, o país com maior número de publicações é os Estados Unidos da América (EUA), com 95 e 102 registros, respectivamente, seguido da China e da Austrália. Observa-se que o tema Adaptabilidade de carreira tem sido tratado em diferentes países, demonstrando a sua importância no mundo todo.

A seguir, apresentam-se os hot topics relacionados à Adaptabilidade de carreira nas bases pesquisadas (Tabelas 5 e 6). Nesta etapa da pesquisa foram elencados os principais tópicos que vêm sendo estudados nos últimos dez anos, relacionados à Adaptabilidade de carreira, conforme pesquisa realizada na literatura, selecionando os temas com maior relação com a temática de estudo. Dessa forma, foram selecionados os 10 tópicos que mais apareceram relacionados à temática, de acordo com o número de publicações, sendo tópicos quentes somente os que possuem o índice $m>2$.

$\mathrm{Na}$ Tabela 5 são apresentados os resultados obtidos das combinações dos temas com o Adaptabilidade de carreira, referentes à base WOS.

Tabela 5

Hot Topics de Adaptabilidade de Carreira na base WoS

\begin{tabular}{|c|c|c|c|c|}
\hline Posição & Tópicos & $\begin{array}{c}\text { Total de } \\
\text { Publicações }\end{array}$ & Índice h-b & Índice $\mathrm{m}$ \\
\hline 1 & $\begin{array}{c}\text { Career development } \\
\text { (Desenvolvimento da carreira) }\end{array}$ & 4.461 & 64 & 6,4 \\
\hline 2 & $\begin{array}{c}\text { Employability } \\
\text { (Empregabilidade) }\end{array}$ & 4.694 & 54 & 5,4 \\
\hline 3 & $\begin{array}{c}\text { Career choices } \\
\text { (Escolha de carreira) }\end{array}$ & 1.104 & 40 & 4,0 \\
\hline 4 & $\begin{array}{c}\text { Career planning } \\
\text { (Planejamento da carreira) }\end{array}$ & 796 & 30 & 3,0 \\
\hline 5 & $\begin{array}{c}\text { Career oppotunities } \\
\text { (Oportunidade de carreira) }\end{array}$ & 785 & 29 & 2,9 \\
\hline 6 & $\begin{array}{l}\text { Career management } \\
\text { (Gestão de carreira) }\end{array}$ & 475 & 26 & 2,6 \\
\hline 7 & $\begin{array}{l}\text { Professional guindance } \\
\text { (Orientação profissional) }\end{array}$ & 308 & 19 & 1,9 \\
\hline 8 & $\begin{array}{l}\text { Career challenges } \\
\text { (Desafios na carreira) }\end{array}$ & 46 & 10 & 1,0 \\
\hline 9 & $\begin{array}{c}\text { Career skills } \\
\text { (Habilidades na carreira) }\end{array}$ & 72 & 7 & 0,7 \\
\hline 10 & $\begin{array}{c}\text { Graduate career } \\
\text { (Pós-graduação na carreira) }\end{array}$ & 46 & 5 & 0,5 \\
\hline
\end{tabular}

Fonte: Web of Science (2020).

Com base na Tabela 5, verifica-se no ranking dos hot topics as combinações dos temas com maior intensidade: Career development (Desenvolvimento da carreira) e Employability (Empregabilidade). Observa-se que na WoS, dos 10 temas, só apareceram 6, sendo que os demais (Professional guindance (Orientação profissional), Career challenges (Desafios na carreira), Career skills (Habilidades na carreira) e Graduate career (pósgraduação na carreira)) apresentaram hot topic $<2.0$.

A Tabela 6 apresenta os hot topics para a base Scopus, relacionados à Adaptabilidade de carreira: 
Luis Felipe Dias Lopes, Adriane Fabricio, Mauren Pimentel Lima e Cristiane Krüger

Tabela 6

Hot topics de Adaptabilidade de Carreira na base Scopus

\begin{tabular}{|c|c|c|c|c|}
\hline Posição & Tópicos & $\begin{array}{c}\text { Total de } \\
\text { Publicações }\end{array}$ & Índice h-b & Índice $\mathrm{m}$ \\
\hline 1 & $\begin{array}{c}\text { Employability } \\
\text { (Empregabilidade) }\end{array}$ & 1.621 & 43 & 4,3 \\
\hline 2 & $\begin{array}{c}\text { Career choices } \\
\text { (Escolhas de carreiras) }\end{array}$ & 736 & 35 & 3,5 \\
\hline 3 & $\begin{array}{c}\text { Career development } \\
\text { (Desenvolvimento da carreira) }\end{array}$ & 934 & 27 & 2,7 \\
\hline 4 & $\begin{array}{l}\text { Career management } \\
\text { (Gestão de carreira) }\end{array}$ & 146 & 18 & 1,8 \\
\hline 5 & $\begin{array}{c}\text { Career planning } \\
\text { (Planejamento de carreira) }\end{array}$ & 150 & 11 & 1,1 \\
\hline 6 & $\begin{array}{l}\text { Career challenges } \\
\text { (Desafios carreira) }\end{array}$ & 20 & 7 & 0,7 \\
\hline 7 & $\begin{array}{c}\text { Career opportunities } \\
\text { (Oportunidade de carreira) }\end{array}$ & 116 & 9 & 0,5 \\
\hline 8 & $\begin{array}{l}\text { Graduate career } \\
\text { (Pós-graduação na carreira) }\end{array}$ & 32 & 5 & 0,5 \\
\hline 9 & $\begin{array}{c}\text { Career Skills } \\
\text { (Habilidades carreira) }\end{array}$ & 14 & 3 & 0,3 \\
\hline 10 & $\begin{array}{l}\text { Professional guindance } \\
\text { (Orientação profissional) }\end{array}$ & $\begin{array}{c}\text { Nenhum } \\
\text { documento }\end{array}$ & --- & --- \\
\hline
\end{tabular}

Fonte: Scopus (2020).

Na Tabela 6, verificam-se três hot topics com maior combinação por intensidade, os quais são Employability (Empregabilidade), Career choices (Escolhas de carreira) e Career development (Desenvolvimento da carreira). Desse modo, é possível identificar que dos temas relacionados à Adaptabilidade de carreira, sete se apresentam abaixo do índice considerado como possíveis temas que possam se tornar relevantes, com índice inferior a 0,5 .

Destaca-se dentre os temas encontrados, que Desenvolvimento de Carreira, Empregabilidade e Escolhas de Carreira foram os três primeiros assuntos em ambas as

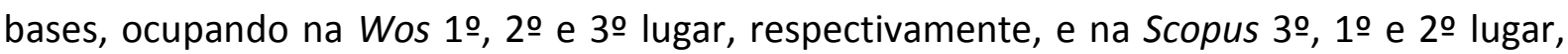
respectivamente, apenas alterando a ordem em cada uma.

De acordo com os resultados obtidos no estudo bibliométrico, é possível verificar que na pesquisa realizada nas bases WoS e Scopus foram encontrados resultados semelhantes sobre a Adaptabilidade de carreira, referentes ao período de 2010 a 2019, demonstrando um aumento gradativo nas publicações.

No Quadro 1 apresenta-se uma síntese dos principais resultados da bibliometria sobre as características básicas e os "hot topics", os quais visam fornecer um panorama básico sobre a temática, auxiliando na melhor visualização e compreensão da Adaptabilidade de carreira.

Quadro 1

Síntese das principais características da bibliometria

\begin{tabular}{|c|c|}
\hline \multicolumn{2}{|c|}{ ADAPTABILIDADE DE CARREIRA } \\
\hline Principais características \\
\hline Web of Science & Scopus \\
\hline Área: Psicologia & Área: Psicologia \\
\hline Ano: 2019 & Ano: 2019 \\
\hline Autor: Yanjun Guan & Autor: Yanjun Guan \\
\hline
\end{tabular}




\begin{tabular}{|c|c|}
\hline Fonte: Journal of vocational behavior & Fonte: Journal of vocational behavior \\
\hline Documento: Artigo & Documento: Artigo \\
\hline País: EUA & País: EUA \\
\hline $\begin{array}{c}\text { Hot Tópics: Carrer development } \\
\text { (Desenvolvimento da carreira) }\end{array}$ & Hot Tópics: Employability \\
(Empregabilidade)
\end{tabular}

Fonte: Web of Science (2020) e Scopus (2020)

Conforme exposto no Quadro 1, observa-se que a principal área para ambas as bases de pesquisa (WoS e Scopus) foi a Psicologia, ligada à área da Saúde. Por ser uma pesquisa que contempla os dez últimos anos, pode-se inferir que os estudos sobre a temática vêm crescendo e torna-se relevante e importante para a academia. Quanto aos autores, percebese que o autor Yanjun Guan se destaca nas duas bases, com 17 publicações.

Por conseguinte, verificou-se que a fonte Journal of Vocational Behavior é a que lidera a pesquisa em Adaptabilidade de carreira, sendo que a maioria das publicações é em forma de artigos. No que se refere aos hot topics, o mais relevante na base WoS é Career development (Desenvolvimento da carreira), enquanto que na base Scopus, é Employability (Empregabilidade).

Destaca-se que, embora as publicações estejam crescendo, conforme apresentados os temas por ano, ainda são poucas na área de Carreira e, principalmente, Adaptabilidade de carreira. Tal fato comprova-se pelas poucas publicações da temática encontradas nas bases de dados Wos e Scopus. Assim, o campo de estudos ainda pode ser muito explorado, a exemplo disso os hot topics < 2.0, mas bem próximos de alcançar esse índice: Professional guindance (Orientação profissional), Career challenges (Desafios na carreira), Career management (Gestão de carreira) e Career planning (Planejamento de carreira).

Na Figura 4 são apresentadas as palavras-chave referentes à análise das publicações encontradas para este estudo bibliométrico, na última década. Assim, realizou-se a identificação das palavras com maior frequência nessas publicações, criando uma nuvem de palavras, a qual foi construída por meio do software Word Cloud.

Figura 4

Palavras chaves da análise bibliométrica sobre Adaptabilidade de carreira

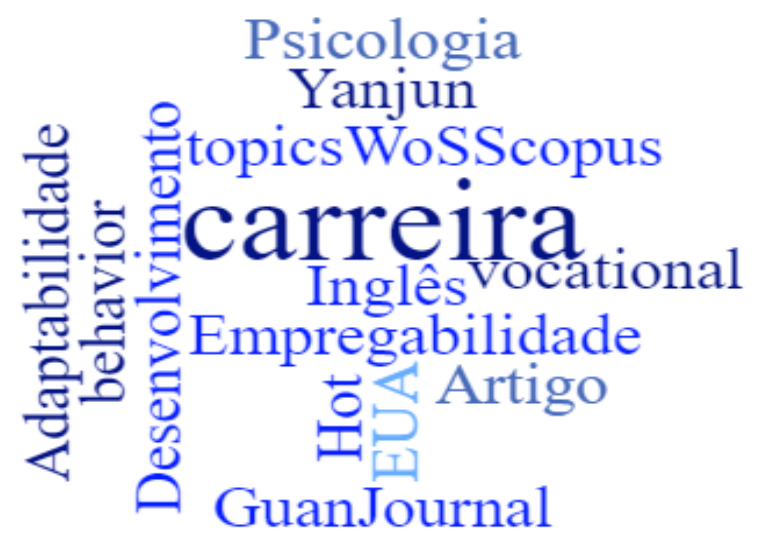

Fonte: Elaborada pelos autores (2020).

A nuvem de palavras apresenta as dimensões das palavras-chave desta pesquisa, as quais se destacam como principais tópicos em cada categoria (ano, área, documento, país e hot topics) relatada. Trata-se de uma ilustração da síntese bibliométrica, a fim de complementar este estudo realizado nas bases WoS e Scopus. 


\section{CONSIDERAÇÕES FINAIS}

O estudo apresentado consistiu em realizar uma pesquisa bibliométrica acerca de Adaptabilidade de carreira. Para tal, foram verificadas as publicações disponíveis nas bases Web of Science e Scopus, relativas ao período de 2010 a 2019, constando 390 e 417 publicações nesse período, respectivamente.

A partir da análise da produção cientifica do tema, observou-se que algumas áreas relacionadas à Adaptabilidade de carreira merecem maior destaque, com base nos resultados obtidos. No que se refere à área temática, percebe-se que o desenvolvimento e adaptação na carreira são os principais fomentadores de publicações de adaptabilidade de carreira.

Quanto ao autor com maior número de registros, percebe-se que este tem como principal interesse de investigação o contexto organizacional e comportamental. Por sua vez, a principal fonte também vai ao encontro das características das áreas temáticas e do principal autor, pois publica pesquisas relacionadas às ciências sociais e comportamentais.

Além disso, foram analisados os hot topics, os temas com maior afinidade e relevantes em Adaptabilidade de carreira, os quais são Desenvolvimento da Carreira e Empregabilidade.

Os resultados obtidos demonstram a expansão da adaptabilidade de carreira nas pesquisas realizadas nas duas bases, WoS e Scopus, as quais ensejam contribuir tanto no âmbito acadêmico como para o empresarial, dada a importância do panorama construído sobre o tema.

Com os avanços teóricos e conceituas, e principalmente em função das novas demandas sociais, provindas durante pandemia COVID-19, o desenvolvimento de carreira e a adaptabilidade de carreira ganharam novos rumos e, com isso, fez com que as pessoas se tornassem mais resilientes, criativas, adaptativas, decisivas e influentes, além de aprenderem a enfrentar novos desafios e, porque não dizer, arriscar novos rumos. A adaptabilidade é um elo de ligação, pois para evoluir profissionalmente podem existir outros meios, outros caminhos, mas é preciso seguir firme no propósito e, principalmente, da forma correta.

Por fim, observou-se que a capacidade de adaptação das pessoas está voltada à empregabilidade, ou seja, primeiro conseguir um emprego e depois manter-se empregado. Isso exige que o trabalhador busque conhecimentos, habilidades e outras características que são valorizadas pelo mercado de trabalho.

Neste intento, o home office vem ensinando os profissionais a serem mais adaptáveis, especialmente em meio a um cenário VUCA (Volatility, Uncertainty, Complexity e Ambiguity), onde é imprescindível estar preparado para as mudanças e para as incertezas. 0 melhor caminho é o aprendizado, manter-se atualizado para novos desafios, através de cursos de qualificação, por exemplo.

Como limitação do estudo, destaca-se a utilização de apenas duas bases de pesquisa de artigos científicos, mesmo que sejam consideradas as principais bases. Assim, sugere-se que estudos futuros ampliem a pesquisa em outras bases de periódicos científicos, bem como em bases de dissertações e teses.

\section{REFERÊNCIAS}


Alves, M. V., \& Miranda, W. C. (2019) O teletrabahlo como meio de flexibilização de direitos trabalhistas após vigência da Lei 13.467/2017. Revista Athenas de Direito, Política e Filosofia, 8(1), p. 1-18.

Banks, M. G. (2006). An extension of the Hirsch index: Indexing scientific topics and compounds. Scientometrics, 69(1), 161-168. https://doi.org/10.1007/s11192-0060146-5

Brasil, V., Felipe, C., Nora, M. M., \& Favreto, R. (2012). Orientação profissional e planejamento de carreira para universitários. Cadernos Acadêmicos, 4(1), 117-131.

Carvalho, T. A. (2007). A escolha e o comprometimento com a Profissão / carreira: um estudo entre psicólogos [Dissertação de Mestrado, Universidade Federal da Bahia]. Repositório da UFBA. https://pospsi.ufba.br/sites/pospsi.ufba.br/files/talyson_amorim.pdf.

Costa, E. G., \& Nebel, L. (2018). O quanto vale a dor? Estudo sobre a saúde mental de estudantes de pós-graduação no Brasil. Revista Polis, Santiago, 17(50), 207-227. http://dx.doi.org/10.4067/S0718-65682018000200207

Dias, M. S. L., \& Soares, D. H. P. (2012). Planejamento de carreira: Uma orientação para universitários. Revista Psicologia Argumento, Curitiba, 30(68), 53-61. https://doi.org/10.7213/psicol.argum.5884

Dutra, J. S. (2017). Gestão de carreiras: a pessoa, a organização e as oportunidades (2a ed.). São Paulo: Atlas.

Dutra, J. S. (2018). Gestão de pessoas: modelos, processos tendências e perspectivas (2a ed.). São Paulo: Atlas.

Ferenhof, H. A., \& Fernandes, R. F. (2015). Passos para construção da revisão sistemática e bibliometria. v. 3.02. https://doi.org/10.13140/RG.2.1.1937.2401/1.

Fiorini, M. C., Bardagi, M. P., \& Silva, N. (2016). Adaptabilidade de carreira: paradigmas do conceito no mundo do trabalho contemporâneo. Revista Psicologia Organizacional do trabalho, 16(3), 236-247. https://doi.org/10.17652/rpot/2016.3.676.

FGV - Fundação Getúlio Vargas (2020). Especialistas debatem transformação da educação em meio a pandemia de COVID-19, Disponível em: https://portal.fgv.br/noticias/especialistas-debatem-transformacao-educacao-meiopandemia-covid-19. Acesso em: 15 de setembro de 2020.

Helal, D. H. (2005, março). Flexibilização organizacional e empregabilidade individual: proposição de um modelo explicativo. Cadernos EBAPE.BR, 3(1), 1-15. https://doi.org/10.1590/S1679-39512005000100006 
Lopes, S., Costa, M. T., Fernández-Llimós, F., Amante, M. J., \& Lopes, P. F. A. (2012). Bibliometria e a avaliação da produção científica: indicadores e ferramentas. In: Actas do congresso Nacional de bibliotecários, arquivistas e documentalistas, 11.

Magalhães, M. O., \& Bendassoli, P. F. (2013). Desenvolvimento de carreiras nas organizações. In: Borges, L. O., \& Mourão, L. (orgs.). O Trabalho e as organizações: atuações a partir da psicologia. Porto Alegre: Artmed, 19(4), 433-460.

Moura, D., Tomei, P. A. (2021). Gestão estratégica de resiliência organizacional (GERO) proposição de framework. Revista Brasileira de Gestão de Negócios, 23(3), 1-21. http://doi.org/ 10.7819/rbgn.v23i3.4118

Nota, L., Ginevra, M. C. \& Soresi, S. (2012). The career and work adaptability questionnaire (CWAQ): A first contribution to its validation. Journal of Adolescence, 35(6), 1557-1569. https://doi.org/10.1016/j.adolescence.2012.06.004

Oliinyk, O., Lehan, I., Sergiienko, L., Kovalenko, L. \& Tarasova, T. (2020). Alternative methodology for assessment of youth competitiveness in the labor market of Ukraine. Management Science Letters, 10(10), 2159-2168. http://doi/org/10.5267/j.msl.2020.3.021

Petska, L. M. (2015). Planejamento e gestão de carreira: um estudo com acadêmicos. [Monografia de Graduação, Centro Universitário Univates]. Repositório da Univates. https://www.univates.br/bdu/bitstream/10737/1020/1/2015LuanaMachadoPestka.pdf

Pimenta, A. A., Portella, A. R. M. R., Oliveira, C. B., \& Ribeiro, R. M. (2017). A bibliometria nas pesquisas acadêmicas. Scientia - Revista de ensino, pesquisa e extensão, 4(7), 1-13.

Savickas, M. L., \& Porfeli, E. J. (2012). Career Adapt-Abilities Scale: Construction, reliability, and measurement equivalence across 13 countries. Journal of Vocational Behavior, 80(3), 661-673. https://doi.org/10.1016/j.jvb.2012.01.011

Souza, R. L., \& Correa, M. A. P. C. (2016). Origem e relação do trabalho com o ser humano e as limitações do trabalho na prisão. Textos \& Contextos, Porto Alegre, 15(1), 126-143. https://doi.org/10.15448/1677-9509.2016.1.22831

Wingerter, D. G., Azevedo, U. N. D., Marcaccini, A. M., Alves, M. D. S. C. F., Ferreira, M. Â. F., \& Miura, L. K. B. (2018). Produção científica sobre quedas e óbitos em idosos: Uma análise bibliométrica. Revista Brasileira de Geriatria e Gerontologia, 21(3), 20-329. https://doi.org/10.1590/1981-22562018021.170168

Zanelli, J. C., Silva, N., \& Tordena, N. (2013). Orientação para aposentadoria e gestão de pessoas nas organizações. In: BORGES, L. O., \& MOURÃO, L. (Orgs.). O Trabalho e as organizações: atuações a partir da psicologia (pp. 644-665). Porto Alegre: Artmed. 